\title{
On the Gibbs Adsorption Equation and Diffuse Interface Models
}

\author{
G. B. McFadden \\ A. A. Wheeler \\ U. S. DEPARTMENT OF COMMERCE \\ Technology Administratıon \\ Mathematical and Computational \\ Sciences Division \\ Natıonal Institute of Standards \\ and Technology \\ Gaithersburg, MD 20899
}

Faculty of Mathematical Studies University of Southampton

Highfield, Southampton So17 1BJ UK

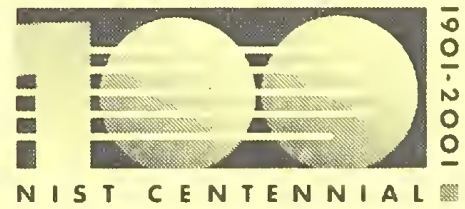





\section{On the Gibbs Adsorption Equation and Diffuse Interface Models}

G. B. McFadden

A. A. Wheeler

U. S. DEPARTMENT OF COMMERCE

Technology Admınıstratıon

Mathematical and Computational

Sciences Division

National Institute of Standards

and Technology

Gaithersburg, MD 20899

Faculty of Mathematıcal Studies

University of Southampton

Highfield, Southampton So17 1BJ UK

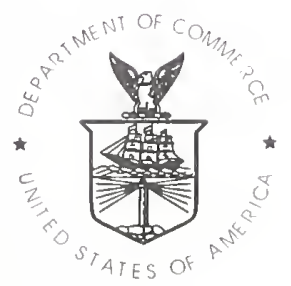

U.S. DEPARTMENT OF COMMERCE Donald L. Evans, Secretary

NATIONAL INSTITUTE OF STANDARDS

AND TECHNOLOGY

Dr. Karen H. Brown, Acting Director 



\title{
On the Gibbs Adsorption Equation and Diffuse Interface Models
}

\author{
G. B. McFadden \\ National Institute of Standards and Technology \\ Gaithersburg, MD 20899-8910, USA \\ and \\ A.A. Wheeler \\ Faculty of Mathematical Studies \\ University of Southampton \\ Highfield, Southampton SO17 1BJ UK
}

\begin{abstract}
In this paper we discuss some applications of the classical Gibbs adsorption equation to specific diffuse interface models that are based on conserved and non-conserved order parameters. Such models are natural examples of the general methodology developed by J.W. Gibbs in his treatment of the thermodynamics of surfaces. We employ the methodology of J.W. Cahn, which avoids the use of conventional dividing surfaces to define surface excess quantities. We show that the Gibbs adsorption equation holds for systems with gradient energy coefficients, provided the appropriate definitions of surface excess quantities are used. We consider in particular the phase field model of a binary alloy with gradient energy coefficients for solute and the phase field. We derive a solute surface excess quantity that is independent of a dividing surface convention, and find that the adsorption in this model is influenced by the surface free energies of the pure components of the binary alloy, as well as the solute gradient energy coefficient. We present one-dimensional numerical solutions for this model corresponding to a stationary planar interface and show the consistency of the numerical results with the Gibbs adsorption equation. We also discuss the Gibbs adsorption equation in the context of other diffuse interface models that arise in spinodal decomposition and order-disorder transitions.
\end{abstract}





\section{Introduction}

Diffuse interface models of phase transitions were first employed to describe a liquid near its critical point. Subsequently, they were developed for a variety of other situations including spinodal decomposition and anti-phase boundaries in solids. The diffuse interface description of an interface envisages that the interface has a small finite thickness over which the material undergoes a smooth transition from one physical state to another, e.g., solid to liquid. The state of the system at each point in space is characterized by one or more order parameters. In an alloy the composition may play the role of an order parameter, which therefore varies continuously within the interfacial region between its value in each of the bulk phases. Consequently other thermodynamic quantities such as the pressure and Helmholtz free-energy density also vary through the interface. The associated variation of the free energy density in the interfacial region gives rise to the surface energy associated with the interface.

Gibbs (1957) in his treatment of interfaces did not explicitly address their internal structure. Rather, he introduced the notion of a dividing surface which separates the two bulk phases and accounts for the physical properties of the interface through additional excess quantities such as composition. In this treatment he derived the Gibbs adsorption equation which relates changes in the surface energy of an interface to corresponding changes in intensive thermodynamic variables, such as temperature and chemical potential, and involves the excess quantities associated with the interface. These ideas have been more recently developed by Cahn (1979).

In this paper we focus on a phase-field model of a binary alloy developed by Wheeler, Boettinger \& McFadden (1993), hereafter denoted by WBM. This model employs a phase-field variable $\phi(\mathbf{x}, t)$ to describe the phase of the system as well as a composition $c(\mathrm{x}, t)$. The governing equations are derived from a free energy functional that contains square gradient terms in both the composition and phase field. It may be shown that for a planar interface in equilibrium the chemical potential is continuous across the interface and the bulk solid and liquid compositions satisfy a common tangent construction. For an ideal solution, this gives a lens-shaped phase diagram. Charach and Fife (1998) have investigated an extensive range of asymptotic limits of this model. Related phase-field models of a simple binary alloy have been also developed by Lin and Rogers (1992), Lowen, Bechhoefer \& Tuckerman (1992), Caginalp \& Xie (1993), Wheeler, Boettinger \& McFadden (1992), Warren \& Boettinger (1995), Kim, Kim \& Suzuki (1999), and Bi \& Sekerka (1998). In addition, phase-field models have been extended to more complex alloys, such as eutectic alloys by Karma (1994) and Wheeler, McFadden \& Boettinger 
996), as well as Steinbach et al. (1996).

Other diffuse models that involve alloy systems include spinodal decomposition (Cahn \& Hilliard 1958) and antiphase boundaries (Allen \& Cahn 1979), as well as multiple order parameter models of order-disorder transitions in body-centered-cubic (BCC) alloys (Cahn \& NovickCohen 1996), face-centered-cubic (FCC) alloys (Braun, Cahn, McFadden \& Wheeler 1997), and hexagonal-close-packed (HCP) alloys (Cahn, Han, \& McFadden 1999).

In this paper we show that the phase-field model of a simple binary alloy satisfies the Gibbs adsorption equation when we make a suitable definition of the excess composition. We present numerical calculations which illustrate that the component of the alloy with the lower surface energy preferentially adsorbs on the interface, as well as how the surface energy depends on both temperature and the solute gradient energy coefficient. We go on to show that the Gibbs adsorption equation may also be derived for other diffuse interface models of alloys, including spinodal decomposition and interphase and antiphase boundaries in a multiple-order-parameter diffuse interface model of order-disorder transitions of an FCC alloy.

\section{The Gibbs-Adsorption Equation for a Binary Alloy}

In this section we review the classical description of the equilibrium surface free energy for a binary alloy, including J.W. Cahn's formulation of the Gibbs adsorption equation. The modifications to this treatment for a phase-field model of a binary alloy are described in the following section.

\subsection{Thermodynamic Equilibrium}

We consider an isothermal binary alloy system comprised of two species, $A$ and $B$. As in our previous treatments (Wheeler et al. 1992, 1993), we assume a constant molar volume throughout the system, which allows us to neglect the effects of volume change on solidification. In this situation the Helmholtz free energy density, $f$, may be considered to depend only on the composition $c$, representing the mole fraction of component $B$, and the temperature of the system, $T$, and satisfies the relation

$$
d f=-s d T+\mu d c
$$


where $s$ is the entropy per unit volume and $\mu=\partial f / \partial c$ is the chemical potential ${ }^{1}$. The pressure $p(c, T)$ is given by

$$
p=-f+c \mu
$$

and satisfies the Gibbs-Duhem equation,

$$
d p=s d T+c d \mu
$$

For equilibrium of a spatially heterogeneous system, these thermodynamic relations are assumed to apply locally at each point, though $f, s, c$, and $p$ may vary from point to point. Gibbs (1957) provides a description of the equilibrium properties of phase boundaries that apply in general, without requiring a detailed model of the interfacial region itself.

We consider a planar solid-liquid interface at temperature $T$ that is at equilibrium, with a semi-infinite solid region extending to $x \rightarrow-\infty$, and a semi-infinite liquid region extending to $x \rightarrow+\infty$. We consider a control volume consisting of a cylinder of uniform cross-sectional area $A$ and length $2 W$ extending over the interval $-W<x<W$. The solid-liquid interface is assumed to be located near $x=0$, and the cylinder is assumed to be long enough that the material near $x=-W$ consists of bulk solid phase and that near $x=W$ is bulk liquid phase. We assume the equilibrium solute profile is described by a function $c(x)$ which tends to bulk equilibrium values in regions far from the solid-liquid interface.

The total Helmholtz free energy of the control volume, $F_{W}$, is given by

$$
F_{W}=A \int_{-W}^{W} f(c, T) d x
$$

and we also define a total entropy $S_{W}$, total composition $C_{W}$, and volume $V_{W}$,

$$
S_{W}=A \int_{-W}^{W} s d x, \quad C_{W}=A \int_{-W}^{W} c d x, \quad V_{W}=A \int_{-W}^{W} d x=2 A W
$$

For a given temperature, $T$, the system within the control volume is at equilibrium if $F_{W}$ is a minimum for given values of $C_{W}$ and $V_{W}$. Introducing constant Lagrange multipliers $\tilde{\mu}$ and $\tilde{p}$,

\footnotetext{
${ }^{1}$ Strictly speaking, a more accurate term for $\mu$ is the diffusion potential, proportional to the difference between the chemical potentials of species $B$ and $A$, but for brevity we will simply refer to $\mu$ as the chemical potential.
} 
the condition for equilibrium is

$$
0=\delta\left\{F_{W}-\tilde{\mu} C_{W}+\tilde{p} V_{W}\right\}
$$

where the minimum is taken with respect to variations in solute, $c(x)+\delta c(x)$, and the end points $x=-W+\delta W_{S}$ and $x=W+\delta W_{L}$, for a fixed value of $A$. This gives

$$
0=A \int_{-W}^{W}\left[f_{c}(c, T)-\tilde{\mu}\right] \delta c d x+\left[f\left(c_{L}, T\right)-\tilde{\mu} c_{L}+\tilde{p}\right] \delta W_{L}-\left[f\left(c_{S}, T\right)-\tilde{\mu} c_{S}+\tilde{p}\right] \delta W_{S}
$$

where $c_{L}=c(W)$ and $c_{S}=c(-W)$ are the equilibrium bulk concentrations in the liquid and solid phases, respectively. The equilibrium concentration profile is thus characterized by a uniform chemical potential. The pressures $p_{L}=c_{L} \mu-f\left(c_{L}, T\right)$ and $p_{S}=c_{S} \mu-f\left(c_{S}, T\right)$ in the bulk liquid and solid phases are equal and satisfy $p_{L}=p_{S}=\tilde{p}$. At equilibrium, then, we have uniform values of $\mu$ and $T$, but only that $p_{L}=p_{S}$ in the bulk phases; $f, c, s$, and $p$ generally vary through the interface.

The equilibrium conditions imply that the common tangent construction holds, as given by

$$
f_{c}\left(c_{L}, T\right)=f_{c}\left(c_{S}, T\right)=\frac{f\left(c_{L}, T\right)-f\left(c_{S}, T\right)}{c_{L}-c_{S}}
$$

These conditions define the liquidus and solidus concentrations, $c_{L}(T)$ and $c_{S}(T)$, as functions of temperature. The chemical potential $\mu(T)$ is also a function of temperature at equilibrium. Prescription of the value of $C_{W}$ serves to specify the position of the interfacial region within the control volume, so that a variation in $C_{W}$ for a given temperature changes the relative amounts of solid and liquid in the control volume.

An explicit example for $f(c, T)$ is provided by the ideal solution model considered in Wheeler et al. (1992), where

$$
f\left(c_{L}, T\right)=f_{L}\left(c_{L}, T\right)=\frac{R T}{v_{m}} I\left(c_{L}\right)
$$

and

$$
f\left(c_{S}, T\right)=f_{S}\left(c_{S}, T\right)=c_{S} \frac{L_{B}\left(T-T_{B}\right)}{T_{B}}+\left(1-c_{S}\right) \frac{L_{A}\left(T-T_{A}\right)}{T_{A}}+\frac{R T}{v_{m}} I\left(c_{S}\right)
$$

where $T_{A}$ and $T_{B}$ are the bulk melting points of components $A$ and $B$, respectively, $L_{A}$ and $L_{B}$ are the latent heats per unit volume of $A$ and $B, R$ is the ideal gas constant, and $v_{m}$ is the 
molar volume, which is assumed to be uniform throughout the system. Here

$$
I(c)=c \ln c+(1-c) \ln (1-c)
$$

is proportional to the ideal entropy of mixing. These free energies lead to a lens-shaped phase diagram, as illustrated in Fig. 1 for a model of the $\mathrm{Cu}$-Ni system with properties listed in Table 1.

\subsection{The Surface Free Energy}

The surface free energy $\gamma_{W}$ can be introduced through the expression

$$
\begin{aligned}
& F_{W}=A \int_{-W}^{W} f(c, T) d x=A \int_{-W}^{W}\left\{\left[f(c, T)-f\left(c_{L}, T\right)\right]-f_{c}\left(c_{L}, T\right)\left[c-c_{L}\right]\right\} d x \\
& +f_{c}\left(c_{L}, T\right) A \int_{-W}^{W} c d x+\left[f\left(c_{L}, T\right)-c_{L} f_{c}\left(c_{L}, T\right)\right] V_{W}=A \gamma_{W}+\mu C_{W}-p_{L} V_{W}
\end{aligned}
$$

where

$$
\gamma_{W}=\int_{-W}^{W}\left\{\left[f(c, T)-f\left(c_{L}, T\right)\right]-f_{c}\left(c_{L}, T\right)\left[c-c_{L}\right]\right\} d x
$$

Using the relation $p=c \mu-f(c, T)$, we may also write

$$
F_{W}=\mu C_{W}-A \int_{-W}^{W} p(x) d x
$$

where the contribution to the free energy from the pressure $p(x)$ appears explicitly. Comparing Eq. (12) and Eq. (14), we see that the surface free energy $\gamma_{W}$ can be interpreted as the free energy associated with the variation of pressure through the interfacial region. We note that $F_{W}, S_{W}$, and $C_{W}$ all vary strongly with the width $W$, since their integrands tend to non-zero limits as $x \rightarrow \pm \infty$. In contrast, $\gamma_{W}$ has a well-defined finite limit for large $W$ given by

$$
\gamma=\int_{-\infty}^{\infty}\left\{\left[f(c, T)-f\left(c_{L}, T\right)\right]-\mu\left[c-c_{L}\right]\right\} d x
$$

where convergence of the integral is made possible by the common tangent construction, Eq. (8), satisfied by the integrand. 


\subsection{The Gibbs Adsorption Equation}

The equilibrium surface energy for a binary alloy can be considered to be a function of $T$ alone, and the Gibbs adsorption equation in this context describes the variation of surface energy with temperature, $d \gamma / d T$. Gibbs' original treatment was described in terms of a dividing surface which is introduced to define surface excess quantities that account for local variation in the interfacial region; for example, a surface excess entropy $S_{x s}$ can be defined by

$$
S_{W}=A \int_{-W}^{W} s(x) d x=s_{S} A \int_{-W}^{X_{0}} d x+s_{L} A \int_{X_{0}}^{W} d x+S_{x s} A
$$

where $x=X_{0}$ is the location of the dividing surface. In this description the bulk entropy densities $s_{L}$ and $s_{S}$ in the liquid and solid are extended to either side of the dividing surface, and the excess quantity $S_{x s}$ is introduced to account for the actual variation of $s(x)$ near the interface. One similarly defines an adsorption, or surface excess concentration, $C_{x s}$, and relates $d \gamma / d T$ to the excess quantities $C_{x s}$ and $S_{x s}$

$$
d \gamma=-S_{x s} d T-C_{x s} d \mu
$$

Although $C_{x s}$ and $S_{x s}$ depend on the location of the dividing surface, the resulting expression for $d \gamma / d T$ does not, consistent with the fact that $d \gamma / d T$ represents a measurable quantity, unlike $S_{x s}$ and $C_{x s}$. We note that $\gamma_{W}$ itself can be interpreted as the surface excess of the quantity $f-c f_{c}=-p$; in this case, however, $\gamma_{W}$ is independent of the location of the dividing surface by virtue of the common tangent construction.

Cahn (1979) gives an elegant discussion of the Gibbs adsorption equation which proceeds without the introduction of a dividing surface. We provide a short summary of Cahn's formulation for the special case of a binary alloy in the next section. This description is then generalized to treat the case of a phase-field model with gradient energy coefficients appearing in the free energy function.

\subsection{Cahn's Formulation of the Gibbs Adsorption Equation}

In terms of the thermodynamic description given in the previous section, if we perturb an equilibrium system that is at temperature $T$ and composition $C_{W}$ by $T \rightarrow T+\delta T$ and $C_{W} \rightarrow$ $C_{W}+\delta C_{W}$, the local composition of the resulting equilibrium state will also be changed, with 
$c(x) \rightarrow c(x)+\delta c(x)$, where

$$
\delta C_{W}=A \int_{-W}^{W} \delta c(x) d x
$$

The variations $\delta T$ and $\delta C_{W}$ represent independent variations; for example, a variation in $C_{W}$ at a given temperature can result from a translation in $x$ of the system that changes the relative amounts of solid and liquid within the control volume.

The corresponding free energy change is given by

$$
\delta F_{W}=A \int_{-W}^{W} \delta f d x=A \int_{-W}^{W}\left[f_{c} \delta c(x)-s \delta T\right] d x=\mu \delta C_{W}-S_{W} \delta T
$$

which is the system-wide version of equation (1). On the other hand, from the relation $F_{W}=$ $A \gamma_{W}-p_{L} V_{W}+\mu C_{W}$ in Eq. (13), a variation at fixed $A$ and $W$ gives

$$
\delta F_{W}=A \delta \gamma_{W}-V_{W} \delta p_{L}+\mu \delta C_{W}+C_{W} \delta \mu
$$

where $\delta \mu$ and $\delta p_{L}$ are the changes in $\mu$ and $p_{L}$ that result from the variations $\delta T$ and $\delta C_{W}$. Comparing Eq. (19) and Eq. (20) leads to the following version of the Gibbs adsorption equation:

$$
A \delta \gamma_{W}=V_{W} \delta p_{L}-C_{W} \delta \mu-S_{W} \delta T
$$

Note, however, that the quantities $V_{W}, C_{W}$, and $S_{W}$ are all strongly-varying functions of the width $W$. To obtain an invariant form for this equation, we follow Cahn's treatment by appending the two Gibbs-Duhem equations for the bulk phases,

$$
\begin{aligned}
& 0=\delta p_{L}-s_{L} \delta T-c_{L} \delta \mu, \\
& 0=\delta p_{S}-s_{S} \delta T-c_{S} \delta \mu,
\end{aligned}
$$

which also represent the increments to Eq. (21) that result from changes of the endpoints at $x=W$ and $x=-W$. If we note that $\delta p_{L}=\delta p_{S}$ at equilibrium, we may then write $(21-23)$ as the linear system

$$
\left(\begin{array}{ccc}
1 & V_{W} & -C_{W} \\
0 & 1 & -c_{L} \\
0 & 1 & -c_{S}
\end{array}\right)\left(\begin{array}{c}
-A \delta \gamma_{W} \\
\delta p_{L} \\
\delta \mu
\end{array}\right)=\left(\begin{array}{c}
S_{W} \\
s_{L} \\
s_{S}
\end{array}\right) \delta T
$$


and use Kramer's rule to solve for $A \delta \gamma_{W}$. This gives

$$
\delta \gamma_{W}=-\delta T \int_{-W}^{W}\left\{\left(s-s_{L}\right)-\frac{\left(s_{S}-s_{L}\right)}{\left(c_{S}-c_{L}\right)}\left(c-c_{L}\right)\right\} d x
$$

This expression has well-defined limits for large $W$, leading to the expression

$$
\begin{aligned}
& \frac{d \gamma}{d T}=-\int_{-\infty}^{\infty}\left\{\left(s-s_{L}\right)-\frac{\left(s_{S}-s_{L}\right)}{\left(c_{S}-c_{L}\right)}\left(c-c_{L}\right)\right\} d x \\
& =-\frac{d \mu}{d T} \int_{-\infty}^{\infty}\left\{\left(c-c_{L}\right)-\frac{\left(c_{S}-c_{L}\right)}{\left(s_{S}-s_{L}\right)}\left(s-s_{L}\right)\right\} d x .
\end{aligned}
$$

The term $\left(s_{S}-s_{L}\right) /\left(c_{S}-c_{L}\right)$ is seen from (22) and (23) to equal $-d \mu / d T$, and Eq. (25) equivalently results from Eq. (21) by using this expression and Eq. (22) to eliminate $p_{L}$. It is also easily seen that the expression (25) is equivalent to the Gibbs adsorption equation (17) by using the appropriate definitions for $d \mu / d T$ and the definitions of the surface excess quantities.

\section{The Phase Field Model for a Binary Alloy}

We next consider the phase-field model of a simple binary alloy discussed by Wheeler et al. (1992), which is based upon a gradient Helmholtz free energy functional given by

$$
F_{W}=\int_{V}\left\{f(c, T, \phi)+\frac{\epsilon^{2}}{2}|\nabla \phi|^{2}+\frac{\kappa^{2}}{2}|\nabla c|^{2}\right\} d V
$$

where $\epsilon$ and $\kappa$ are constant gradient energy coefficients; we denote the solute gradient energy coefficient by $\kappa$ rather than $\delta$, the more common notation, to avoid confusion with variational quantities. This model has also been studied by a number of other authors as well, including Charach \& Fife $(1998,1999)$ and Cahn \& Novick-Cohen $(2000)$.

The free energy density considered by Wheeler et al. (1992) is given by an ideal solution model

$$
f(c, T, \phi)=r(\phi) f_{S}(c, T)+[1-r(\phi)] f_{L}(c, T)+\frac{W(c)}{4} g(\phi),
$$

where $W(c)=c W_{B}+(1-c) W_{A}$ is a linear combination of the double well heights for the pure components, and

$$
g(\phi)=\phi^{2}\left(1-\phi^{2}\right), \quad r(\phi)=\phi^{2}(3-2 \phi) .
$$

The phase-field variable $\phi$, which labels the liquid and solid phases in our model, is analogous 
to an order parameter in solid-state order-disorder transitions (Allen \& Cahn 1979). In this context, the liquid phase in our model is analogous to a disordered phase with $\phi=0$, and the solid phase is analogous to the ordered phase with $\phi=1$. The phase-field variable can also be interpreted in terms of the density of atoms in the solid-liquid system. In the solid phase, the density varies periodically on the scale of the crystal lattice, whereas the mean density is uniform in the liquid phase, with a smooth transition between the two states in the interfacial region. The phase-field variable can then be regarded as characterizing the amplitude of the density modulation. With the convention that $\phi=0$ represents the liquid phase and $\phi=1$ represents the solid phase, the above functions $g(\phi)$ and $r(\phi)$ satisfy $g(0)=g(1)=0, r(0)=0$, and $r(1)=1$, resulting in $f(c, T, 1)=f_{S}(c, T)$ and $f(c, T, 0)=f_{L}(c, T)$.

An equivalent expression for the free energy density is

$$
f(c, T, \phi)=c f_{B}(\phi, T)+[1-c] f_{A}(\phi, T)+\frac{R T}{v_{m}} I(c),
$$

where $I(c)$ is given in Eq. (11). Here $f_{A}$ and $f_{B}$ are the free energy densities of components $A$ $(c=0)$ and $B(c=1)$, respectively, and are given by

$$
f_{A}(\phi, T)=\frac{L_{A}\left(T-T_{A}\right)}{T_{A}} r(\phi)+\frac{W_{A}}{4} g(\phi), \quad f_{B}(\phi, T)=\frac{L_{B}\left(T-T_{B}\right)}{T_{B}} r(\phi)+\frac{W_{B}}{4} g(\phi),
$$

cf. Eq. (10). At the melting points of the pure components, the equilibrium surface energies and widths of the solid-liquid interface can be related to the double well heights and gradient energy coefficient, viz. (Wheeler et al. 1992)

$$
\gamma_{A}=\frac{\epsilon}{6} \sqrt{W_{A} / 2}, \quad \gamma_{B}=\frac{\epsilon}{6} \sqrt{W_{B} / 2}
$$

and

$$
\ell_{A}=\frac{\epsilon}{\sqrt{W_{A} / 2}}, \quad \ell_{B}=\frac{\epsilon}{\sqrt{W_{B} / 2}}
$$

\subsection{Thermodynamic Equilibrium for the Phase-Field Model}

Equilibrium inside the control volume of length $2 W$ is described by the Euler equations

$$
\kappa^{2} c_{x x}=f_{c}(c, T, \phi)-\tilde{\mu}
$$




$$
\epsilon^{2} \phi_{x x}=f_{\phi}(c, T, \phi)
$$

where $\tilde{\mu}$ is again a Lagrange multiplier representing the constraint on the total concentration $C_{W}$, which is defined by Eq. (5) in this case as well. Neumann boundary conditions $c_{x}(W)=$ $c_{x}(-W)=0$ and $\phi_{x}(W)=\phi_{x}(-W)=0$ arise naturally if the energy is minimized in the control volume with respect to $c$ and $\phi$; if the width $W$ is large enough compared to the interfacial region, the solute and phase fields will be uniform far from the interfacial region. In that case the generalized chemical potential $f_{c}-\kappa^{2} c_{x x}=\tilde{\mu}$ reduces to the classical chemical potential in the bulk solid and liquid phases.

The Euler equations admit a first integral

$$
\frac{\epsilon^{2}}{2} \phi_{x}^{2}+\frac{\kappa^{2}}{2} c_{x}^{2}=\left[f(c, T, \phi)-f\left(c_{L}, T, 0\right)\right]-\tilde{\mu}\left[c-c_{L}\right]
$$

and by examining the far field values of equations (34) and (36) we see that the common tangent conditions (8) relating $c_{L}, c_{S}$, and $T$ also apply in this case.

\subsection{Surface Free Energy for the Phase-Field Model}

The surface free energy in the phase-field model includes the gradient energy terms, and is defined by writing

$$
\begin{gathered}
F_{W}=A \int_{-W}^{W}\left\{f(c, T, \phi)+\frac{\epsilon^{2}}{2} \phi_{x}^{2}+\frac{\kappa^{2}}{2} c_{x}^{2}\right\} d x \\
=A \int_{-W}^{W}\left\{\frac{\epsilon^{2}}{2} \phi_{x}^{2}+\frac{\kappa^{2}}{2} c_{x}^{2}+\left[f(c, T, \phi)-f\left(c_{L}, T, 0\right)\right]-f_{c}\left(c_{L}, T, 0\right)\left[c-c_{L}\right]\right\} d x \\
+f_{c}\left(c_{L}, T, 0\right) A \int_{-W}^{W} c d x+\left[f\left(c_{L}, T, 0\right)-c_{L} f_{c}\left(c_{L}, T, 0\right)\right] V_{W} \\
=A \gamma_{W}+\tilde{\mu} C_{W}-p_{L} V_{W},
\end{gathered}
$$

where we have assumed that $c_{x x}(W)$ is negligible in replacing $f_{c}\left(c_{L}, T, 0\right)$ by $\tilde{\mu}$ in the last equation. This expression has the same form as in Eq. (12), but now instead of the definition (13) we have

$$
\gamma_{W}=\int_{-W}^{W}\left\{\frac{\epsilon^{2}}{2} \phi_{x}^{2}+\frac{\kappa^{2}}{2} c_{x}^{2}+\left[f(c, T, \phi)-f\left(c_{L}, T, 0\right)\right]-f_{c}\left(c_{L}, T, 0\right)\left[c-c_{L}\right]\right\} d x
$$




\subsection{Gibbs Adsorption Equation for the Phase-Field Model}

A derivation for the Gibbs adsorption relation corresponding to this phase-field model follows the same lines as given above; for brevity we simply compute $d \gamma / d T$ by varying the temperature only. A variation in temperature causes variations in $\gamma_{W}, \tilde{\mu}$ and $p_{L}$, as well as the functions $c(x)$ and $\phi(x)$. From Eq. (37) we have

$$
\frac{d F_{W}}{d T}=A \frac{d \gamma_{W}}{d T}+\frac{d \tilde{\mu}}{d T} C_{W}+\tilde{\mu} \frac{d C_{W}}{d T}-\frac{d p_{L}}{d T} V_{W}
$$

We also have

$$
\begin{gathered}
\frac{d F_{W}}{d T}=A \frac{d}{d T} \int_{-W}^{W}\left\{f(c, T, \phi)+\frac{\epsilon^{2}}{2} \phi_{x}^{2}+\frac{\kappa^{2}}{2} c_{x}^{2}\right\} d x \\
=A \int_{-W}^{W}\left\{f_{c} c_{T}+f_{T}+f_{\phi} \phi_{T}+\epsilon^{2} \phi_{x} \phi_{x T}+\kappa^{2} c_{x} c_{x T}\right\} d x \\
=A \int_{-W}^{W}\left\{\left(f_{c}-\kappa^{2} c_{x x}\right) c_{T}-s+\left(f_{\phi}-\epsilon^{2} \phi_{x x}\right) \phi_{T}\right\} d x, \\
=\tilde{\mu} \frac{d C_{W}}{d T}-S_{W},
\end{gathered}
$$

where we have integrated by parts and used the Euler equations to obtain the final expression. Comparing Eq. (39) and Eq. (40) gives

$$
\frac{d \gamma_{W}}{d T}=-\frac{d \tilde{\mu}}{d T} C_{W}-S_{W}+\frac{d p_{L}}{d T} V_{W}
$$

Using

$$
\frac{d p_{L}}{d T}=s_{L}+c_{L} \frac{d \mu}{d T}
$$

and

$$
\frac{d p_{S}}{d T}=s_{S}+c_{S} \frac{d \mu}{d T}
$$

and following Cahn's treatment as before, leads again to the expression

$$
\frac{d \gamma_{W}}{d T}=-\frac{d \mu}{d T} \int_{-W}^{W}\left\{\left(c-c_{L}\right)-\frac{\left(c_{S}-c_{L}\right)}{\left(s_{S}-s_{L}\right)}\left(s-s_{L}\right)\right\} d x
$$

thus the addition of gradient energy terms does not alter the form (25) of the classical Gibbs adsorption equation. However, the gradient terms do affect the profiles that comprise the integrands in (44) as will be illustrated numerically in a specific example below. 


\section{Numerical Calculations}

In this section we describe numerical calculations for the model binary alloy considered by Wheeler et al. (1992). Numerical solutions were computed by discretizing the Euler equations (34) and (35) and using second-order-accurate finite differences on a uniform grid of $2 N+1$ points, with a mesh spacing of $h=W / N$. Neumann boundary conditions were applied at $x= \pm W$, resulting in $2 N+4$ nonlinear equations in the $2 N+4$ unknowns $c_{j} \approx c(j h)$ and $\phi_{3} \approx \phi(j h)$., for $j=-N, \ldots, N$. In addition, the Lagrange multiplier $\tilde{\mu}$ is computed by specifying a value for the total solute $C_{W}$ : An alternative possibility is to specify the condition $\phi(0)=1 / 2$ instead of the solute constraint; both conditions serve to locate the position of the interfacial region inside the box. The resulting set of nonlinear equations is solved using the package SNSQ (Powell 1970). Acceptable accuracy is obtained using 200 grid points provided that the width $W$ is an order of magnitude wider than the interfacial region.

\subsection{The Phase-Field Model without Solute Gradient Energy Terms $(\kappa=0)$}

We first set $\kappa=0$ and study the effect on interface adsorption of varying the surface free energies of the pure components, $\gamma_{A}$ and $\gamma_{B}$. Some interface profiles for $T=1700 \mathrm{~K}$ are shown in Fig. 2. The dashed curve corresponds to the choice $\gamma_{A}=\gamma_{B}$; Table 2 contains the resulting values of $\gamma$, normalized by $\gamma_{A}$, and the quantity $d \gamma / d T$, normalized by $\gamma_{A} / T_{A}$. This temperature is near the pure A side of the phase diagram, and the concentrations and surface free energies are near those of component A.

If $\gamma_{A}$ is fixed and $\gamma_{B}$ is increased (lower curves), the equilibrium profile is characterized by depletion of $B$ in the interfacial region, consistent with the minimization of the free energy by reducing the amount of the higher-energy component in the interface. The surface free energy increases with increasing $\gamma_{B}$, and $d \gamma / d T$ becomes increasingly negative.

If $\gamma_{B}$ is fixed and $\gamma_{A}$ is increased (upper curves), the situation is reversed, and the lowest energy state has preferential incorporation of component $B$ in the interfacial region, which is also reflected in the trends shown in Table 2.

We next show in Fig. 3 the dependence of $\gamma$ on temperature for $\gamma_{A}=\gamma_{B}=3.7\left(10^{-5}\right)$ $\mathrm{J} / \mathrm{cm}^{2}$. Although the surface free energy of the pure components are equal, the surface energy is larger for intermediate values of the system temperature, reflecting the influence of the solute 
contribution to the surface free energy.

The corresponding surface adsorption is shown in Fig. 4. Here we define a dimensionless surface adsorption

$$
\Gamma_{x s}=\frac{1}{2 l_{A}} \int_{-W}^{W}\left\{\left(c-c_{L}\right)-\frac{\left(c_{S}-c_{L}\right)}{\left(s_{S}-s_{L}\right)}\left(s-s_{L}\right)\right\} d x
$$

The derivative of the surface free energy $\gamma_{W}$ with respect to temperature is proportional to $-\Gamma_{x s} d \mu / d T$. The 's-shaped' adsorption curve reflects the change in sign of $d \gamma / d T$, which is nonmonotonic in this case. We emphasize that $\Gamma_{x s}$ is independent of a dividing surface convention, and generalizes the adsorption coefficient that was displayed in Fig. 3 of WBM; $\Gamma_{x s}$ includes contributions from both the solute and the entropy profiles.

We next show in Fig. 5 the dependence of $\gamma$ on temperature for $\gamma_{A}=3.7\left(10^{-5}\right) \mathrm{J} / \mathrm{cm}^{2}$ and $\gamma_{B}=2.8\left(10^{-5)} \mathrm{J} / \mathrm{cm}^{2}\right.$. In this case the surface free energy is a monotonic function of temperature, as the contribution to the surface free energy from solute is less than the variation that is associated with the different values for the pure components.

The corresponding adsorption is shown in Fig. 6. The adsorption $\Gamma_{x s}$ is positive, with a maximum at an intermediate temperature near the middle of the phase diagram.

\subsection{The Phase-Field Model with Solute Gradient Energy Terms $(\kappa \neq$ 0)}

We next present numerical calculations with a non-zero solute gradient energy coefficient $\kappa$. We take $\gamma_{A}=3.7\left(10^{-5}\right) \mathrm{J} / \mathrm{cm}^{2}$ and $\gamma_{B}=2.8\left(10^{-5}\right) \mathrm{J} / \mathrm{cm}^{2}$, with $\epsilon=3.3\left(10^{-6}\right)(\mathrm{J} / \mathrm{cm})^{1 / 2}$; the case $\kappa=0$ therefore corresponds to Figs. 5 and 6. In Fig. 7 we show the surface free energy for $\kappa=\epsilon=3.3\left(10^{-6}\right)(\mathrm{J} / \mathrm{cm})^{1 / 2}, \kappa=1.0\left(10^{-5}\right)(\mathrm{J} / \mathrm{cm})^{1 / 2}, \kappa=2.0\left(10^{-5}\right)(\mathrm{J} / \mathrm{cm})^{1 / 2}$, and $\kappa=3.3\left(10^{-5}\right)(\mathrm{J} / \mathrm{cm})^{1 / 2}$. The curve for $\kappa=\epsilon=3.3\left(10^{-6}\right)(\mathrm{J} / \mathrm{cm})^{1 / 2}$, indicated by a dashed line, is almost indistinguishable from the case $\kappa=0$ shown in Fig. 5. As $\kappa$ increases, the surface free energy also increases due to the enhancement of the free energy from the solute gradient energy contribution. For the value $\kappa=3.3\left(10^{-5}\right)(\mathrm{J} / \mathrm{cm})^{1 / 2}$, the maximum surface free energy occurs at an intermediate temperature in the interior of the phase diagram. The corresponding adsorption coefficients are shown in Fig. 8. For larger values of $\kappa$, the adsorption adopts an 's-shaped' profile with both positive and negative values, which is consistent with the observations in Fig. 3 of WBM, which corresponded to a limiting case $\epsilon / \kappa \ll 1$ (in WBM, $\kappa$ was 
denoted by $\delta$ ). For $\epsilon / \kappa \ll 1$ the width of the transition layer for the solute profile is much larger than that for the phase field, as discussed in WBM and Charach \& Fife (1999). The surface free energy curve shown in Fig. 4 of WBM for $\kappa=3.3\left(10^{-5}\right) \mathrm{J} / \mathrm{cm}^{1 / 2}$ shows a non-monotonic temperature dependence that is similar to the present calculations with $\epsilon=3.3\left(10^{-6}\right) \mathrm{J} / \mathrm{cm}^{1 / 2}$ and $\kappa=3.3\left(10^{-5}\right) \mathrm{J} / \mathrm{cm}^{1 / 2}$.

\section{Discussion}

We next mention some variations of this procedure, which apply to special cases and generalizations of the solidification model that we have discussed above. We start with two examples, given by the Cahn-Hilliard equation and the binary alloy model of Kim, Kim, and Suzuki (1999), where analytic solutions allow the Gibbs adsorption equation to be verified explicitly. We then discuss an example with multiple order parameters that illustrates the dependence of the Gibbs adsorption equation on the thermodynamic degrees of freedom in the system.

\subsection{Cahn-Hilliard Equation}

The Cahn-Hilliard equation is a special case of the binary alloy phase-field model that results from setting $\epsilon=0$ and taking a special form of the free energy density that is independent of カ. A simple model for spinodal decomposition near the critical temperature $T_{\mathrm{cr}}$ and critical mposition $c_{\mathrm{cr}}$ is obtained by assuming

$$
f(c, T)=a\left(T-T_{\mathrm{cr}}\right)\left(c-c_{\mathrm{cr}}\right)^{2}+b\left(c-c_{\mathrm{cr}}\right)^{4},
$$

where $a$ and $b$ are positive constants. If $T<T_{\mathrm{cr}}$, a solute profile of the form

$$
c(x)=c_{\mathrm{cr}}+\left[\frac{a\left(T_{\mathrm{cr}}-T\right)}{2 b}\right]^{1 / 2} \tanh \frac{x}{2 \xi}
$$

satisfies the Cahn-Hilliard equation, where the width $\xi$ of the interface is given by

$$
\xi^{2}=\frac{\kappa^{2}}{4 a\left(T_{\mathrm{cr}}-T\right)}
$$


The surface energy of the interface is given by

$$
\gamma(T)=\int_{-\infty}^{\infty}\left\{\frac{\kappa^{2}}{2} c_{x}^{2}+f(c, T)\right\} d x=\frac{2 \kappa a^{3 / 2}\left(T_{\mathrm{cr}}-T\right)^{3 / 2}}{3 b} .
$$

This model is symmetric about the point $c=c_{\mathrm{cr}}$, so that the chemical potential $\mu$ is zero for the planar system for all $T$. The resulting form of the Gibbs adsorption equation,

$$
\frac{d \gamma}{d T}=-\int_{-\infty}^{\infty}[s(x)-s(\infty)] d x=\frac{-\kappa a^{3 / 2}\left(T_{\mathrm{cr}}-T\right)^{1 / 2}}{b}
$$

can be verified directly, where the entropy density is $s=-f_{T}=-a\left(c-c_{\mathrm{cr}}\right)^{2}$.

\subsection{Kim, Kim, and Suzuki's Model of a Binary Alloy}

A model for the solidification of a binary alloy that has a very simple description of the equilibrium phase field and solute profiles across the solid-liquid interface was given by Kim, Kim, and Suzuki (1999). In their model, $\phi$ is interpreted as a solid fraction, and solute concentrations $c_{L}(x)$ and $c_{S}(x)$ in the liquid and solid are defined throughout the system; the solute concentration at each point in space is then given by

$$
c=r(\phi) c_{S}+[1-r(\phi)] c_{L}
$$

where $r(\phi)$ is a smooth function of $\phi$ with $r(0)=0$ and $r(1)=1$; e.g., $r(\phi)=\phi$ or $r(\phi)=$ $\phi^{2}(3-2 \phi)$. The concentrations $c_{L}(x)$ and $c_{S}(x)$ are also assumed to satisfy a parallel tangent construction at each point, with

$$
\frac{\partial f_{L}\left(c_{L}, T\right)}{\partial c_{L}}=\frac{\partial f_{S}\left(c_{S}, T\right)}{\partial c_{S}}
$$

where $f_{L}$ and $f_{S}$ are the prescribed bulk free energy densities in each phase. Eqns. (51) and (52) are inverted to give the relations $c_{L}=c_{L}(c, T, \phi)$ and $c_{S}(c, T, \phi)$, and the free energy density $f(c, T, \phi)$ is then defined as

$$
f(c, T, \phi)=r(\phi) f_{S}\left(c_{S}(c, T, \phi)\right)+[1-r(\phi)] f_{L}\left(c_{L}(c, T, \phi)\right)+\frac{W(T)}{4} g(\phi)
$$

which differs from the form used in Eq. (28) in that $c_{S}$ and $c_{L}$ appear in the arguments of $f_{S}$ and $f_{L}$ rather than the concentration $c$ itself. Kim, Kim, and Suzuki (1999) assume a constant 
double well height $W$, but we assume here that the height depends on temperature as a means of allowing different surface free energies at $T=T_{A}$ and $T=T_{B}$.

The free energy functional of the system has the form (27) with $\kappa=0$, and the equilibrium equations

$$
f_{c}=\tilde{\mu}, \quad f_{\phi}-\epsilon^{2} \phi_{x x}=0
$$

have particularly simple solutions because of the specific form chosen for $f(c, T, \phi)$. By using (51) and (52) we find that $f_{\phi}(c, T, \phi)=W(T) g^{\prime}(\phi) / 4$, so that the phase field is given by the profile

$\phi(x)=[1-\tanh (x / 2 \ell)] / 2$, where $\ell(T)=\epsilon / \sqrt{W(T)}$. In addition, the equilibrium equations imply that the common tangent construction holds, which in this case results in $c_{L}(x)$ and $c_{S}(x)$ being constants that are given by the liquidus and solidus values at the given temperature $T$; the solute profile $c(x)$ in $(51)$ is then simply an interpolation by $r(\phi)$ between the liquidus and solidus values.

The derivative of the surface free energy is

$$
\begin{gathered}
\frac{d \gamma}{d T}=\frac{d}{d T} \int_{-\infty}^{\infty}\left\{\frac{\epsilon^{2}}{2} \phi_{x}^{2}+\frac{W(T)}{4} g(\phi)\right\} d x \\
=\frac{W^{\prime}(T)}{4} \int_{-\infty}^{\infty} g(\phi) d x+\int_{-\infty}^{\infty}\left\{\epsilon^{2} \phi_{x} \phi_{x T}+\frac{W}{4} g^{\prime}(\phi) \phi_{T}\right\} d x=\frac{W^{\prime}(T)}{4} \int_{-\infty}^{\infty} g(\phi) d x
\end{gathered}
$$

where an integration by parts again simplifies the final expression. On the other hand, we have

$$
s(x)=-f_{T}=r(\phi) s_{S}+[1-r(\phi)] s_{L}-\frac{W^{\prime}(T)}{4} g(\phi)
$$

where $s_{S}=-\partial f_{S}\left(c_{S}, T\right) / \partial T$ and $s_{L}=-\partial f_{L}\left(c_{L}, T\right) / \partial T$ are the bulk entropy densities in each phase, so that the Gibbs adsorption equation gives

$$
\frac{d \gamma}{d T}=-\int_{-\infty}^{\infty}\left\{\left[s(x)-s_{L}\right]-\frac{\left[s_{S}-s_{L}\right]}{\left[c_{S}-c_{L}\right]}\left[c(x)-c_{L}\right]\right\} d x=\frac{W^{\prime}(T)}{4} \int_{-\infty}^{\infty} g(\phi) d x
$$

in agreement with (55).

\subsection{Diffuse Interface Model of FCC Alloys}

Adsorption also arises in models of interphase boundaries (IPBs) and antiphase boundaries (APBs) that are based on diffuse interface descriptions; examples include face-centered-cubic alloys (Braun et al. 1997) and hexagonal-close-packed alloys (Cahn, Han, \& McFadden 1999). In 
numerical computations for an FCC system performed by Tonaglu (2000), adsorption is observed in the concentration profiles associated with APBs. This model provides a good illustration of the appropriate degrees of freedom that appear in the Gibbs adsorption equation, and we provide a brief summary of the governing equations here.

The FCC model is based on four order parameters that describe how the two species are distributed on the FCC lattice, corresponding to the corners and faces of the unit cell. The overall concentration in the system is denoted by $c$, a conserved order parameter, and there are, in addition, three non-conserved order parameters, denoted by $X, Y$, and $Z$. The disordered phase is characterized by $X=Y=Z=0$, and ordered states have one or more non-zero values of $X, Y$, and $Z$. There is an associated free energy density $f(c, X, Y, Z, T)$. The free energy functional for the system includes three gradient energy coefficients $A, B$, and $\kappa$, and takes the form

$$
\begin{aligned}
F=\int_{V}\left\{\frac { A } { 2 } \left(X_{x}^{2}\right.\right. & \left.+Y_{y}^{2}+Z_{z}^{2}\right)+\frac{B}{2}\left(X_{y}^{2}+X_{z}^{2}+Y_{x}^{2}+Y_{z}^{2}+Z_{x}^{2}+Z_{y}^{2}\right) \\
& \left.+\frac{\kappa^{2}}{2}|\nabla c|^{2}+f(c, X, Y, Z, T)\right\} d V
\end{aligned}
$$

For a planar system with unit normal $\vec{n}=\left(n_{x}, n_{y}, n_{z}\right)$, a one-dimensional solution depends only on the variable $\zeta=\vec{n} \cdot(x, y, z)$ that measures distance from the interface. The system has the associated free energy

$$
F_{W}=A \int_{-W}^{W}\left\{\frac{\alpha_{x}^{2}}{2} X_{\zeta}^{2}+\frac{\alpha_{y}^{2}}{2} Y_{\zeta}^{2}+\frac{\alpha_{z}^{2}}{2} Z_{\zeta}^{2}+\frac{\kappa^{2}}{2} c_{\zeta}^{2}+f(c, X, Y, Z, T)\right\} d \zeta,
$$

where

$$
\alpha_{x}^{2}=A n_{x}^{2}+B n_{y}^{2}+B n_{z}^{2}, \quad \alpha_{y}^{2}=B n_{x}^{2}+A n_{y}^{2}+B n_{z}^{2}, \quad \alpha_{z}^{2}=B n_{x}^{2}+B n_{y}^{2}+A n_{z}^{2} .
$$

The Euler equations are

$$
f_{c}-\kappa^{2} c_{\zeta \zeta}=\tilde{\mu}
$$

where the Lagrange multiplier $\tilde{\mu}$ again is equal to the chemical potential, and the non-conserved order parameters satisfy

$$
\alpha_{x}^{2} X_{\zeta \zeta}-f_{X}=0, \quad \alpha_{y}^{2} Y_{\zeta \zeta}-f_{Y}=0, \quad \alpha_{z}^{2} Z_{\zeta \zeta}-f_{Z}=0
$$


A first integral of the system is given by

$$
\begin{gathered}
0=\frac{\alpha_{x}^{2}}{2} X_{\zeta}^{2}+\frac{\alpha_{y}^{2}}{2} Y_{\zeta}^{2}+\frac{\alpha_{z}^{2}}{2} Z_{\zeta}^{2}+\frac{\kappa^{2}}{2} c_{\zeta}^{2} \\
+[f(c, X, Y, Z, T)-f(c(\infty), X(\infty), Y(\infty), Z(\infty), T)]-\tilde{\mu}[c-c(\infty)] .
\end{gathered}
$$

\subsubsection{Interphase Boundaries}

An IPB connecting the disordered bulk phase with concentration $c_{d i s}$ and an ordered bulk phase with concentration $c_{o r}$ and associated order parameters $\left(X_{0}, Y_{0}, Z_{0}\right) \neq(0,0,0)$ is described by the above Euler equations, with solutions that satisfy the far-field boundary conditions

$$
[c(\zeta), X(\zeta), Y(\zeta), Z(\zeta)] \rightarrow\left\{\begin{array}{ccc}
{\left[c_{o r}, X_{0}, Y_{0}, Z_{0}\right]} & \text { as } & \zeta \rightarrow-\infty \\
{\left[c_{d i s}, 0,0,0\right]} & \text { as } & \zeta \rightarrow \infty
\end{array}\right.
$$

say. The situation is analogous to our previous treatment of the binary alloy solidification problem, with the disordered phase playing the role of the liquid phase, and the ordered phase

playing the role of the ordered phase. The Euler equations again imply that the common tangent construction holds, which in this case is given by

$$
f_{c}\left(c_{d i s}, 0,0,0, T\right)=f_{c}\left(c_{o r}, X_{0}, Y_{0}, Z_{0}, T\right)=\frac{f\left(c_{d i s}, 0,0,0, T\right)-f\left(c_{o r}, X_{0}, Y_{0}, Z_{0}, T\right)}{c_{d i s}-c_{o r}}=\tilde{\mu}
$$

and

$$
\begin{gathered}
f_{X}\left(c_{d i s}, 0,0,0, T\right)=f_{Y}\left(c_{d i s}, 0,0,0, T\right)=f_{Z}\left(c_{d i s}, 0,0,0, T\right)=0, \\
f_{X}\left(c_{o r}, X_{0}, Y_{0}, Z_{0}, T\right)=f_{Y}\left(c_{o r}, X_{0}, Y_{0}, Z_{0}, T\right)=f_{Z}\left(c_{o r}, X_{0}, Y_{0}, Z_{0}, T\right)=0 .
\end{gathered}
$$

Employing a control volume of uniform cross-sectional area $A$ as before, extending over the interval $-W<\zeta<W$ in this case, leads to a corresponding surface free energy $\gamma_{W}$ given by

$$
\begin{gathered}
\gamma_{W}=\int_{-W}^{W}\left\{\frac{\kappa^{2}}{2} c_{\zeta}^{2}+\frac{\alpha_{x}^{2}}{2} X_{\zeta}^{2}+\frac{\alpha_{y}^{2}}{2} Y_{\zeta}^{2}+\frac{\alpha_{z}^{2}}{2} Z_{\zeta}^{2}\right. \\
\left.+\left[f(c, X, Y, Z, T)-f\left(c_{d i s}, 0,0,0, T\right)\right]-\tilde{\mu}\left[c-c_{d i s}\right]\right\} d \zeta,
\end{gathered}
$$

in terms of which we have

$$
F_{W}=A \gamma_{W}+\tilde{\mu} C_{W}-p_{d i s} V_{W}
$$


where $p_{d i s}$, the pressure in the bulk disordered phase, is given by $p_{d i s}=c_{d i s} f_{c}\left(c_{d i s}, 0,0,0, T\right)-$ $f\left(c_{d i s}, 0,0,0, T\right)$. The bulk equilibrium concentrations are then functions of the temperature, $c_{d i s}(T)$ and $c_{o r}(T)$, whose loci form curves in the phase diagram analogous to the liquidus and solidus curves in the solidification case. The temperature is the sole thermodynamic degree of freedom for an IPB, and the Gibbs adsorption equation

$$
\frac{d \gamma_{W}}{d T}=-\int_{-W}^{W}\left\{\left(s-s_{d i s}\right)-\frac{\left(s_{o r}-s_{d i s}\right)}{\left(c_{o r}-c_{d i s}\right)}\left(c-c_{d i s}\right)\right\} d \zeta
$$

for this diffuse interface system can be derived as before, in this case by making use of the Euler equations (61) and (62) to simplify the result. Here the entropy density is given by $s=-f_{T}(c, X, Y, Z, T)$.

\subsubsection{Antiphase Boundaries}

An APB connects variants of an ordered bulk phase. The variants have the same bulk concentrations $c_{o r}$ and free energy; but the composition and temperature of the ordered phase are independent thermodynamic degrees of freedom, in contrast to the case of an IPB. The nonconserved order parameters assume different values in the two variants; examples include an APB connecting two $\mathrm{L} 1_{0}$ variants with $X=X_{0} \neq 0, Y=Z=0$ and $X=-X_{0}, Y=Z=0$, or an APB connecting two $\mathrm{L}_{2}$ variants with $X=Y=Z=X_{0} \neq 0$ and $X=-Y=-Z=X_{0}$.

The boundary conditions for the Euler equations (61) and (62) in this case are $c \rightarrow c_{o r}$ as $\zeta \rightarrow \pm \infty$; the far-field values of the non-conserved order parameters are denoted by $X(\infty)$, $X(-\infty)$, etc. The variants have equal energy,

$$
f\left(c_{o r}, X(-\infty), Y(-\infty), Z(-\infty), T\right)=f\left(c_{o r}, X(\infty), Y(\infty), Z(\infty), T\right)
$$

which follows from the first integral (63) for this case.

The surface adsorption and surface excess entropy for the APB are well-defined quantities, with no need for a dividing surface convention, and are given by

$$
C_{x s}=\int_{-\infty}^{\infty}\left(c-c_{o r}\right) d \zeta, \quad S_{x s}=\int_{-\infty}^{\infty}\left(s-s_{o r}\right) d \zeta
$$

where $s_{o r}=-f_{T}\left(c_{o r}, X(\infty), Y(\infty), Z(\infty), T\right)$ is the entropy density of the bulk ordered phase. 
In the control volume we define the surface free energy $\gamma_{W}$ by

$$
\begin{aligned}
\gamma_{W}= & \int_{-W}^{W}\left\{\frac{\kappa^{2}}{2} c_{\zeta}^{2}+\frac{\alpha_{x}^{2}}{2} X_{\zeta}^{2}+\frac{\alpha_{y}^{2}}{2} Y_{\zeta}^{2}+\frac{\alpha_{z}^{2}}{2} Z_{\zeta}^{2}\right. \\
& \left.+\left[f(c, X, Y, Z, T)-f\left(c_{o r}, X(\infty), Y(\infty), Z(\infty), T\right)\right]-\tilde{\mu}\left[c-c_{o r}\right]\right\} d \zeta .
\end{aligned}
$$

For a fixed control volume, we consider changes $\delta \gamma_{W}$ due to variations $\delta T$ in temperature and $\delta c_{o r}$ in the bulk composition; there are also associated changes in the local profiles of the concentration and the non-conserved order parameters, as well as in the total concentration $C_{W}$, the chemical potential $\tilde{\mu}$, and the bulk pressure $p_{o r}$. The variations $\delta C_{W}$ and $\delta c(x)$ are related as in Eq. (18). Note that a translation of the system within the control volume does not cause an appreciable change in $C_{W}$ provided $W$ is large enough; therefore we do not assume that $\delta C_{W}$ can be varied independently of $T$ and $c_{o r}$.

The free energy of the control volume is $F_{W}=A \gamma_{W}+\tilde{\mu} C_{W}-p_{o r} V_{W}$, and examination of its variation again leads to the familiar equation

$$
A \delta \gamma_{W}=V_{W} \delta p_{o r}-C_{W} \delta \tilde{\mu}-S_{W} \delta T
$$

where the Euler equations can be used to eliminate the contributions from the gradient energy terms as before. For an APB there is a single Gibbs-Duhem equation that holds in the bulk for both variants,

$$
\delta p_{o r}=s_{o r} \delta T+c_{o r} \delta \tilde{\mu}
$$

which leads to the result

$$
\delta \gamma_{W}=-\delta T \int_{-W}^{W}\left[s(x)-s_{o r}\right] d \zeta-\delta \tilde{\mu} \int_{-W}^{W}\left[c(x)-c_{o r}\right] d \zeta=-S_{x s} \delta T-C_{x s} \delta \tilde{\mu} .
$$

Here

$$
\delta \tilde{\mu}=f_{c c}\left(c_{o r}, X(\infty), Y(\infty), Z(\infty), T\right) \delta c_{o r}+f_{c T}\left(c_{o r}, X(\infty), Y(\infty), Z(\infty), T\right) \delta T
$$

which allows the calculation of $d \gamma_{W} / d T$ and $d \gamma_{W} / d c_{o r}$ directly in terms of $S_{x s}, C_{x s}$, and second derivatives of the free energy density. 


\section{Conclusion}

In this paper we have shown that diffuse interface models of alloy systems satisfy the Gibbs adsorption equation. In particular, we have investigated in detail a phase-field model of a binary alloy involving gradient energy terms in both the phase-field and composition. Numerical solutions of a planar interface using this model exhibit the component with the lower surface energy preferentially adsorbing onto the interface as well as the surface energy being enhanced through the presence of the solute gradient energy term. We also considered examples using models of spinodal decomposition and order-disorder transitions in binary alloys. In each case we have verified that the Gibbs adsorption equation holds for systems that feature gradient energy coefficients and combinations of conserved and non-conserved order parameters, provided that the appropriate definitions of surface excess quantities are used.

\section{Acknowledgments}

This work was conducted with the support of the Microgravity Research Division of the National Aeronautics and Space Administration. We thank W.J. Boettinger, J.W. Cahn, S.R. Coriell, K.F. Gurski, and R.F. Sekerka for helpful discussions.

\section{References}

[1] Allen, S.M. \& Cahn, J.W. 1979 A microscopic theory for antiphase motion and its application to antiphase domain coarsening. Acta Metall. 27. 1085-1095.

[2] Bi Z.Q. \& Sekerka, R.F. 1998 Phase-field model of solidification of a binary alloy, Phys. Rev. A 261, 95-106.

[3] Braun, R.J.,Cahn, J.W., McFadden,G.B. \& Wheeler A.A. 1997 Anisotropy of interfaces in an ordered alloy: a multiple-order-parameter model. Phil. Trans. Roy. Soc. Lond. A 355, 1787-1833.

[4] Caginalp, G \& Xie, W. 1993 Phase-field and sharp interface models. Phys. Rev. E 48, 1897-1909.

[5] Cahn, J.W. 1979 Thermodynamics of solid and fluid surfaces. In Interfacial Segregation, (ed. W.C. Johnson \& J.M. Blakely), pp. 3-23. Metals Park, OH: ASM. 
[6] Cahn, J.W., Han, S.C., \& McFadden, G.B. 1999 Anisotropy of interfaces in an ordered HCP binary alloy. Journal of Statistical Physics 95, 1337-1360.

[7] Cahn, J.W. and Hilliard, J.E. 1958 Free energy of a nonuniform system: I. Interfacial free energy. J. Chem. Phys. 28, 258-267.

[8] Cahn, J.W. \& Novick-Cohen, A. 1996 Evolution equations for phase separation and ordering in binary alloys. Journal of Statistical Physics 76 877-909.

[9] Cahn, J.W. \& Novick-Cohen, A. 2000 Motion by curvature and impurity drag: resolution of a mobility paradox. Acta Mater. 48 3425-3440.

[10] Charach, C \& Fife, P.C. 1998 Solidification fronts and solute trapping in a binary alloy. SIAM J. Applied Math. 58, 1826-51.

[11] Charach, C. \& Fife, P.C. 1999 Phase field models of solidification in binary alloys: capillarity and solute trapping effects. J. Crystal Growth 199, 1267-1274.

[12] Conti, M. 1997 Solidification of binary alloys: thermal effects studied with the phase-field model. Phy. Rev. E 54, 588-599.

[13] Gibbs, J.W. 1957 Collected Works, Vol. I (Yale University Press, New Haven), 219-331.

[14] Karma, A. 1994 Phase-field model of eutectic growth. Phs. rev. E 49, 2245-2250.

[15] Kim, S.G., Kim, W.T., \& Suzuki, T. 1999 Phase field model for binary alloys. 60, 7186-7197.

[16] Lin. T. \& Rogers, R.C. 1995 On an order-parameter model for a binary liquid. Computational Materials Science 4, 159-171.

[17] Lowen, H., Bechhoefer, J. \& Tuckerman, L. S. 1992 Crystal growth at long times: critical behavior at the crossover from diffusion to kinetics-limited regimes. Phys. Rev. A 45, 23992415.

[18] Powell, M.J.D. 1970 A hybrid method for nonlinear equations. In Numerical Methods for Nonlinear Algebraic Equations, (ed. P. Rabinowitz), pp. 87-161. Gordon \& Breach: New York.703. 
[19] Steinbach, I., Pezzolla, F., Nestler, B., Seeselberg, M., Prieler, R., Schmitz, G. J. \& Rezenda, J.L.L. 1996 A phase field concept for multiphase systems. Physica D 94 135147.

[20] Tanoglu, G.B. 2000 Phase boundaries and anisotropy via multiple-order-parameter theory for an FCC alloy. Ph.D. Dissertation, Department of Mathematics, University of Delaware, Newark, Delaware.

[21] Warren, J.A. and Boettinger, W.J. 1995 Prediction of dendritic growth and microsegregation patterns in a binary alloy using the phase field method. Acta Metall. Mater. 43 689-703.

[22] Wheeler, A.A., Boettinger, W.J., \& McFadden, G.B. 1992 Phase-field model for isothermal phase transitions in binary alloys. Phys. Rev. A 45 7424-7439.

[23] Wheeler, A.A., Boettinger, W.J., \& McFadden, G.B. 1993 Phase-field model of solute trapping. Phys. Rev. B 47 1893-1909.

[24] Wheeler, A. A., McFadden, G. B. \& Boettinger, W. J. 1996 Phase-field model for solidification of a eutectic alloy. Proc. R. Soc. Lond. A 452, 495-525. 


\begin{tabular}{|cc|}
\hline$L_{A}$ & $2350 \mathrm{~J} / \mathrm{cm}^{3}$ \\
$L_{B}$ & $1725 \mathrm{~J} / \mathrm{cm}^{3}$ \\
$T_{A}$ & $1728 \mathrm{~K}$ \\
$T_{B}$ & $1358 \mathrm{~K}$ \\
$v_{m}$ & $7.4 \mathrm{~cm}^{3} /$ mole \\
$R$ & $8.314 \mathrm{~J} / \mathrm{mole} \mathrm{K}$ \\
\hline
\end{tabular}

Table 1: Material parameters used in phase diagram calculations.

\begin{tabular}{|cccc|}
\hline$\gamma_{A}\left(\mathrm{~J} / \mathrm{cm}^{2}\right)$ & $\gamma_{B}\left(\mathrm{~J} / \mathrm{cm}^{2}\right)$ & $\gamma / \gamma_{A}$ & $\left(T_{A} / \gamma_{A}\right) d \gamma / d T$ \\
\hline $11.2\left(10^{-5}\right)$ & $2.8\left(10^{-5}\right)$ & 0.952673 & 2.9194647 \\
$8.4\left(10^{-5}\right)$ & $2.8\left(10^{-5}\right)$ & 0.959877 & 2.4917755 \\
$5.6\left(10^{-5}\right)$ & $2.8\left(10^{-5}\right)$ & 0.969577 & 1.8930718 \\
$2.8\left(10^{-5}\right)$ & $2.8\left(10^{-5}\right)$ & 1.006204 & -0.363581 \\
$2.8\left(10^{-5}\right)$ & $5.6\left(10^{-5}\right)$ & 1.115590 & -6.733671 \\
$2.8\left(10^{-5}\right)$ & $8.4\left(10^{-5}\right)$ & 1.261922 & -14.490109 \\
$2.8\left(10^{-5}\right)$ & $11.2\left(10^{-5}\right)$ & 1.417201 & -22.055166 \\
\hline
\end{tabular}

Table 2: Material parameters used in adsorption calculations. Here $T=1700$ $\mathrm{K}, \epsilon=3.3\left(10^{-6}\right)(\mathrm{J} / \mathrm{cm})^{1 / 2}, l_{A}=\epsilon^{2} / 6 \gamma_{A}$.

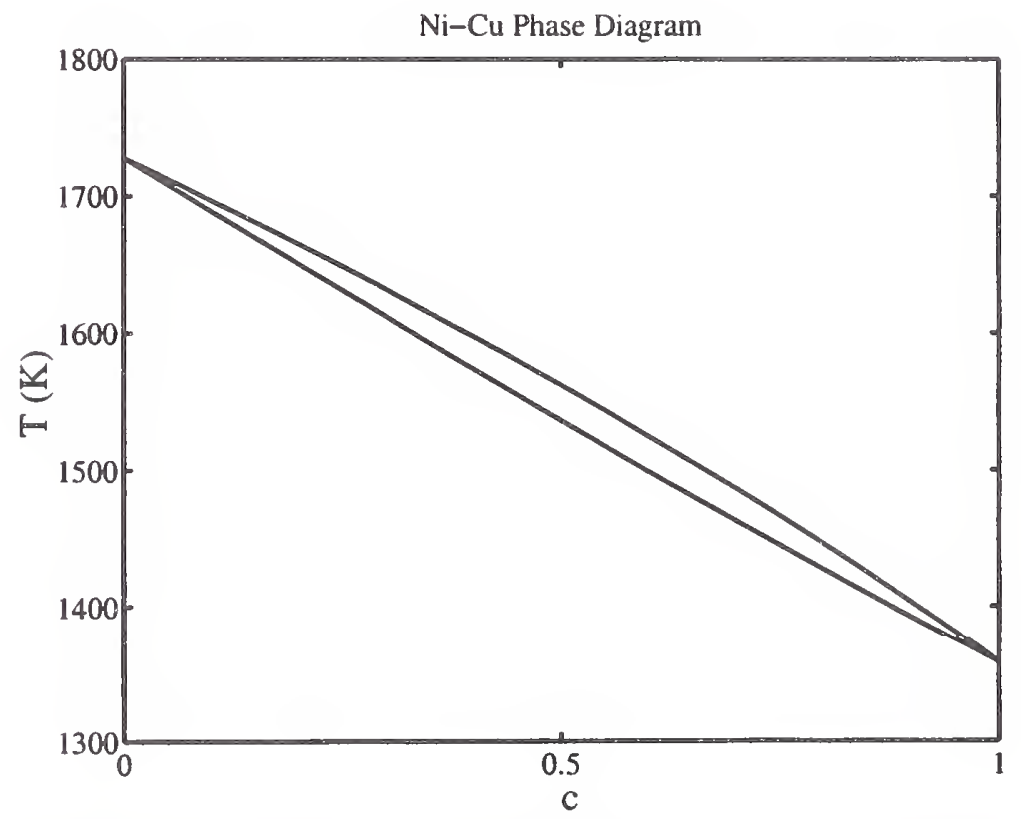

Figure 1: Phase diagram using the parameters in Table 1. 


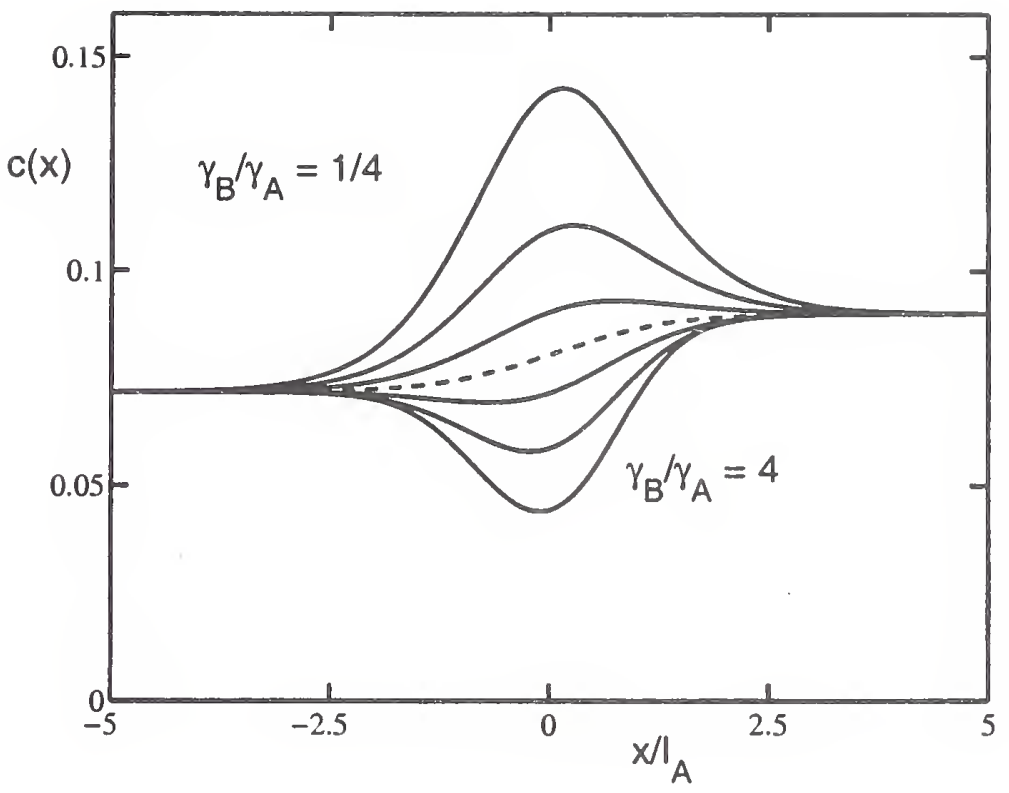

Figure 2: Concentration profiles using the parameters in Table 2.

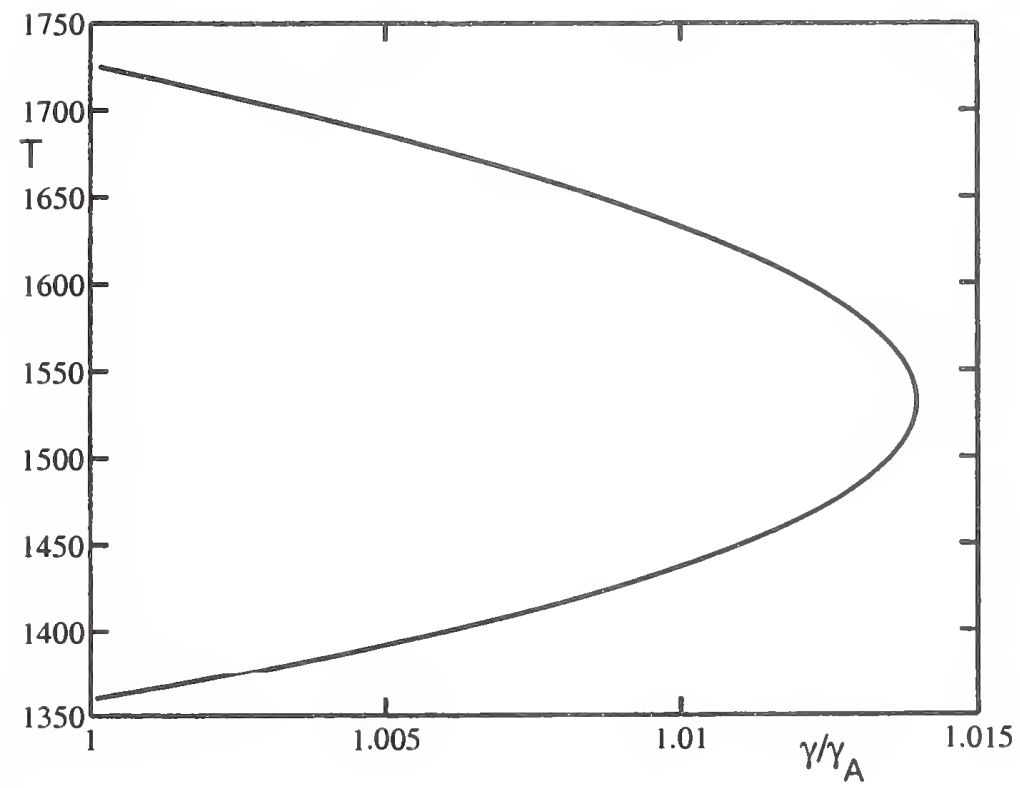

Figure 3: The dependence of $\gamma$ on temperature for $\gamma_{A}=\gamma_{B}=3.7\left(10^{-5}\right) \mathrm{J} / \mathrm{cm}^{2}$. 


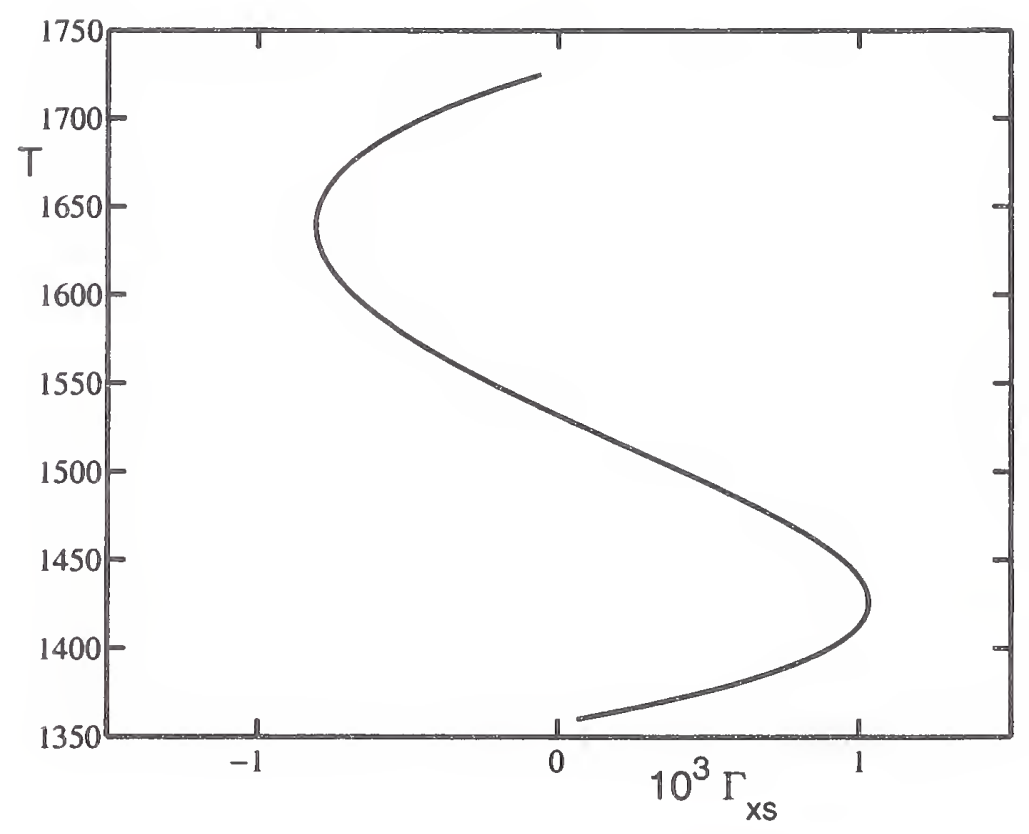

Figure 4: The dependence of the dimensionless surface adsorption $\Gamma_{x s}$ on temperature for $\gamma_{A}=\gamma_{B}=3.7\left(10^{-5}\right) \mathrm{J} / \mathrm{cm}^{2}$.

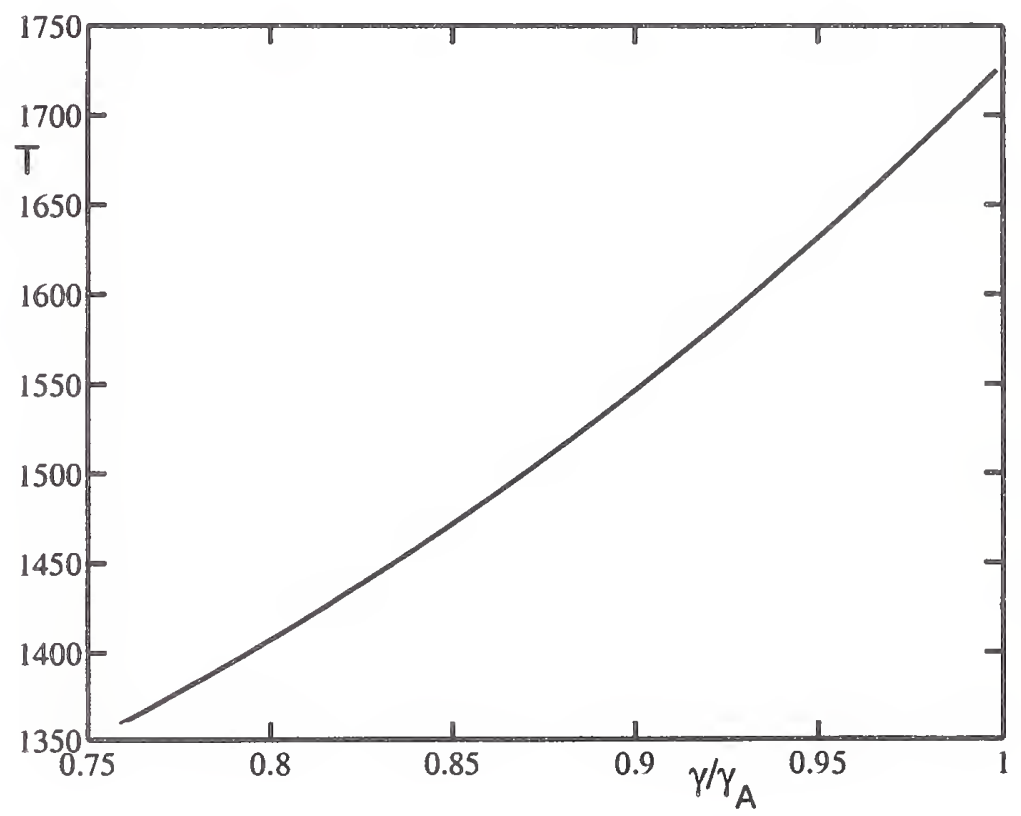

Figure 5: The dependence of $\gamma$ on temperature for $\gamma_{A}=3.7\left(10^{-5}\right) \mathrm{J} / \mathrm{cm}^{2}$ and $\gamma_{B}=2.8\left(10^{-5}\right) \mathrm{J} / \mathrm{cm}^{2}$. 


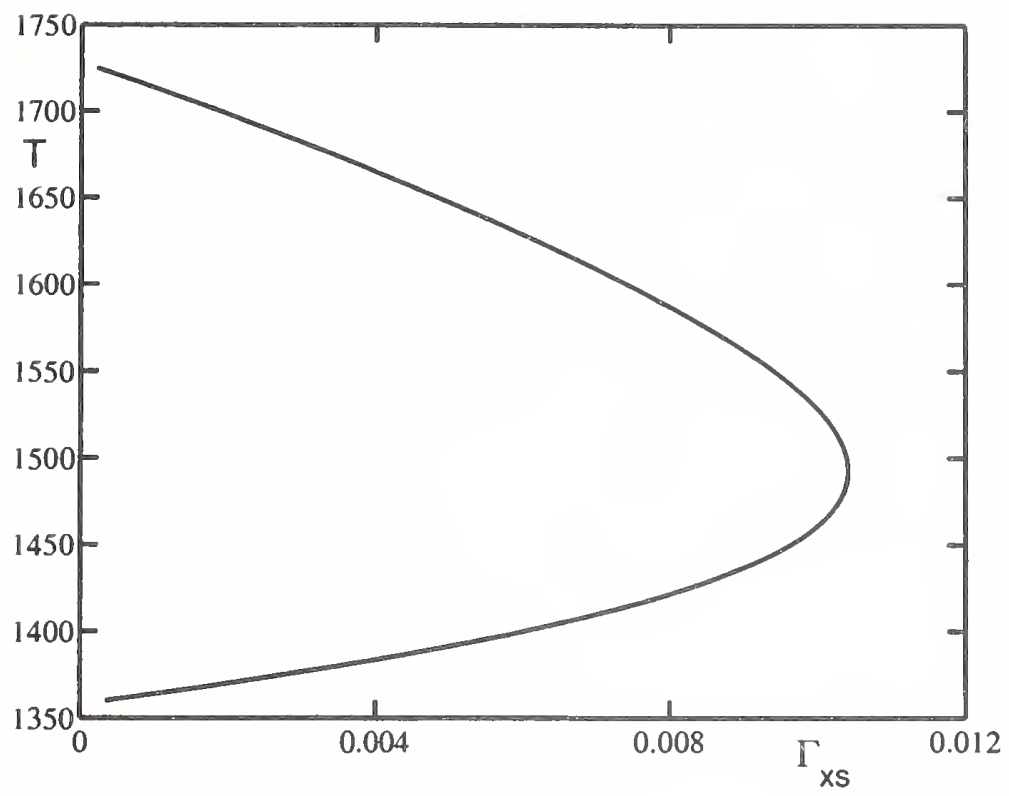

Figure 6: The dependence of the dimensionless surface adsorption $\Gamma_{x s}$ on temperature for $\gamma_{A}=3.7\left(10^{-5}\right) \mathrm{J} / \mathrm{cm}^{2}$ and $\gamma_{B}=2.8\left(10^{-5}\right) \mathrm{J} / \mathrm{cm}^{2}$.

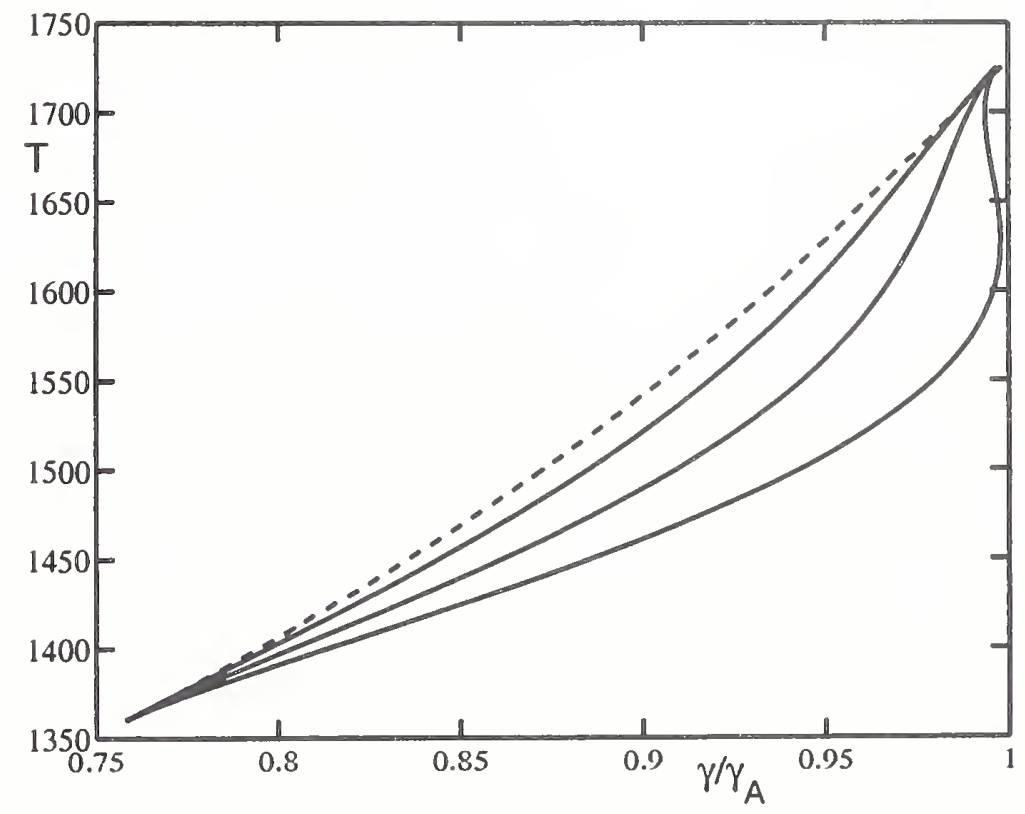

Figure 7: The surface free energy for $\kappa=\epsilon=3.3\left(10^{-6}\right)(\mathrm{J} / \mathrm{cm})^{1 / 2}$ [dashed curve], $\kappa=1.0\left(10^{-5}\right)(\mathrm{J} / \mathrm{cm})^{1 / 2}, \kappa=2.0\left(10^{-5}\right)(\mathrm{J} / \mathrm{cm})^{1 / 2}$, and $\kappa=3.3\left(10^{-5}\right)$ $(J / \mathrm{cm})^{1 / 2}$. 


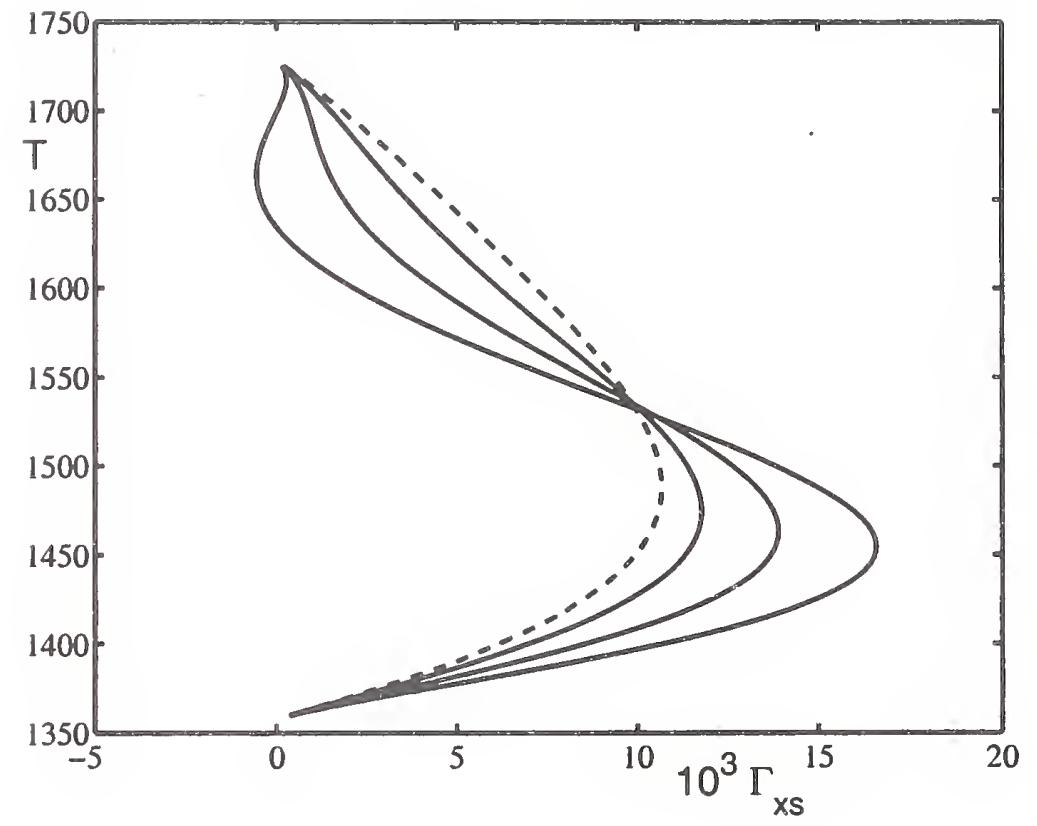

Figure 8: The surface adsorption for $\kappa=\epsilon=3.3\left(10^{-6}\right)(\mathrm{J} / \mathrm{cm})^{1 / 2}$ [dashed curve], $\kappa=1.0\left(10^{-5}\right)(\mathrm{J} / \mathrm{cm})^{1 / 2}, \kappa=2.0\left(10^{-5}\right)(\mathrm{J} / \mathrm{cm})^{1 / 2}$, and $\kappa=3.3\left(10^{-5}\right)$ $(\mathrm{J} / \mathrm{cm})^{1 / 2}$. 

